\title{
Numerical Simulation on Inertial Measurement Unit Errors
}

\author{
P. SUN, G.D. LI, Z.L. ZHANG \& X.J. WANG \\ Wuhan Ordnance Non-Commissioned Officer Academy, Wuhan, China
}

\begin{abstract}
In this paper, a systematic numerical analysis on strap-down inertial measurement unit errors is developed for performance validation. An inertial measurement unit (IMU) incorporates various errors that will have influence on its performance. Understanding these errors is the first step to accurately accounting for them and obtaining an accurate estimate of the state of the vehicles on which the IMU is mounted. This paper attempts to characterize the total error in the measurement of the IMU as a result of the contribution of various IMU error sources. Error models for IMU bias, scale factor, misalignment errors, and random errors are given. The effect of these errors on the measurement of the IMU is simulated. The results demonstrate the validity of the models.
\end{abstract}

KEYWORD: Inertial Measurement Unit (IMU); Error Modeling; Simulation

\section{INTRODUCTION}

Due to its small size, high precision, good concealment, the strap-down inertial measurement unit system has developed rapidly in military and civilian fields in recent years.[1] The strap-down inertial measurement unit often consists of three orthogonally mounted accelerometers and three orthogonally mounted gyros.[2] From a survey of the literature, it emerges that most of the calibration procedures proposed for IMUs were based on a numerical analysis on the errors and the modeled IMU errors are mainly sensor biases. So this paper attempts to characterize the total error in the measurement of the IMU to form an essential component of the construction of strap-down navigation system accuracy validation.

This paper is outlined as follows. In Section 2, the various reference frames used are firstly summarized. IMU measurements and errors are discussed in details in Section 3. Section 4 focus on numerical simulation results and finally a conclusion is given in Section 5.

\section{REFERENCE FRAMES}

Coordinate frames related in the case study of initial alignment of the IMU are illustrated as Figure 1. Inertial frame (i), navigation frame (t), body frame (b) and earth fixed frame (e) are used in this paper. Refer to [3] for the details of these frames.

\section{IMU MEASUREMENTS AND ERRORS}

The performance of IMU is affected by various errors. The IMU acceleration or angular velocity measurements are usually biased from the correct value.[4] Zero drift bias start constant and stay that way, while non-zero drift might increase with time. Scale factor relates to IMU linearity and might have an error which either linearly or non-linearly. Misalignment error is about the mounting orthogonality of IMU axes. The IMU measurement noise is composed of white noise, correlated noise, random walk, quantization errors and dither noise.

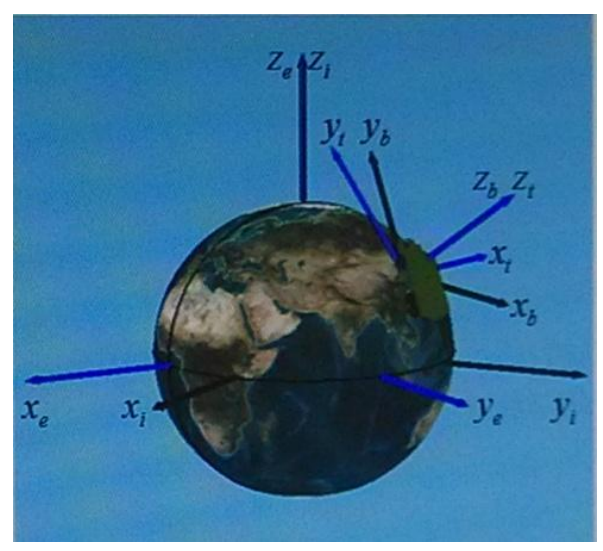

Figure1. Coordinate frames

\subsection{Measurement model}

Measurement model of accelerometer is as follows:[5] 
$m_{a}=f+b_{a}+S_{1} f+S_{2}(f)+N f+e_{a}$

where $m_{a}$ is the measurement vector given by IMU; $f$ describes specific force; $b_{a}$ is the bias error of measurement; $S_{1}$ is the linear scale factor errors; $\mathrm{S}_{2}(\mathrm{f})$ represents the non-linear scale factor error; $\mathrm{N}$ is the misalignment matrix; $\varepsilon_{\mathrm{a}}$ is the noise vector.

\subsection{Noise}

Variance of the noise vector $\varepsilon a$ can be described as follows:

$$
\begin{aligned}
& \sigma^{2}(\tau)=\sigma_{N}^{2}(\tau)+\sigma_{B}^{2}(\tau)+\sigma_{K}^{2}(\tau)+\sigma_{R}^{2}(\tau)+ \\
& \sigma_{Q}^{2}(\tau)+\sigma_{M}^{2}(\tau)+\sigma_{s}^{2}(\tau)
\end{aligned}
$$

which is composed of white noise, correlated noise, random walk, quantization error, and dither noise. Least square method can be used to get the value of the parameters:

$$
\begin{aligned}
& \sigma_{\Omega}^{2}(\tau)=\frac{R^{2}}{2} \tau^{2}+\frac{K^{2}}{3} \tau+\frac{2 B^{2} \ln 2}{\pi}+N^{2} \tau^{-1}+ \\
& 3 Q^{2} \tau^{-2}=\sum_{n=-2}^{2} C_{n} \tau^{n}
\end{aligned}
$$

where $\mathrm{k}=1,2,3, \cdots_{\mathrm{kmax}} \leq 0.5(\mathrm{M}-1)$.

\subsection{Bias}

The bias error ba is composed of a bias at normal operating conditions and an added drift. According to [6], bias changes as a function of temperature:

$$
\begin{aligned}
& \Delta b=0.2\left(\frac{T-T_{\min }}{T_{k a l}-T_{\min }}\right) \\
& \dot{\mathbf{b}}_{\mathbf{a}}=\mathbf{w}+\Delta \dot{\mathbf{b}}, \mathbf{b}_{\mathbf{a}}(0)=\mathbf{b}_{\mathbf{0}}, \mathbf{w} \in N\left(0, \mathbf{b}_{\mathbf{1}}^{2}\right)
\end{aligned}
$$

where $\mathrm{b}_{0}$ is the zero $\mathrm{g}$ bias, $\Delta \mathrm{b}$ is the added bias, $\mathrm{w}$ is a white Gaussian noise that drives the bias to drift, and $b_{1}$ is the drift white noise standard deviation.

\subsection{Misalignment error}

Package misalignment error is defined as the angle between the true axis of sensitivity and the body axis of the IMU. Figure 2 shows the rotation about $\mathrm{z}, \mathrm{y}$ and $\mathrm{x}$ axis.

\subsection{Scale factor error}

The scale factor error is a kind of non-linearity error given as a percentage from the measurement full scale.[7]

The angular velocity measurement of the gyroscopes is modeled using the following equation:

$$
m_{w}=w+b_{w}+S_{w} w+S_{2 w}(w)+N_{w} w+e_{w}
$$

where $\mathrm{m}_{\omega}$ is the gyroscope IMU measurement; $\omega$ is the true angular velocity vector; $b_{\omega}$ is the gyro measurement bias error. The gyroscope measurement noise $\varepsilon_{\omega}$ is composed of white noise, correlated noise, random walk, quantization error, and dither noise.

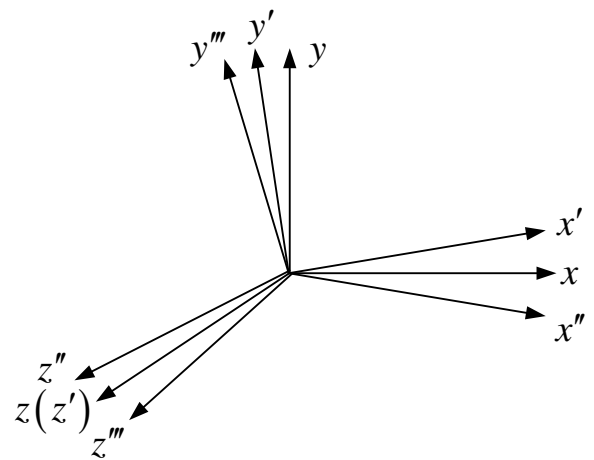

Figure2. Rotation about three axes

\section{SIMULATIONS}

The contribution of errors to the measurement of IMU is simulated with the profile that an IMU is of zero-truth angular velocity and specific force. In this simulation, IMU errors are caused by the bias error and added white measurement noise. The zero-bias of each gyroscope is set as $0.02 \mathrm{deg} / \mathrm{s}$ and the zerobias of each accelerometer is set as $0.2 \mathrm{~m} / \mathrm{s}^{2}$. A measurement noise standard deviation of 0.0003 and 0.002 was used for the gyroscopes and the accelerometers, respectively. The gyroscopes' measurements errors are shown in Figure 3 and the accelerometers' measurements errors are shown in Figure 4.

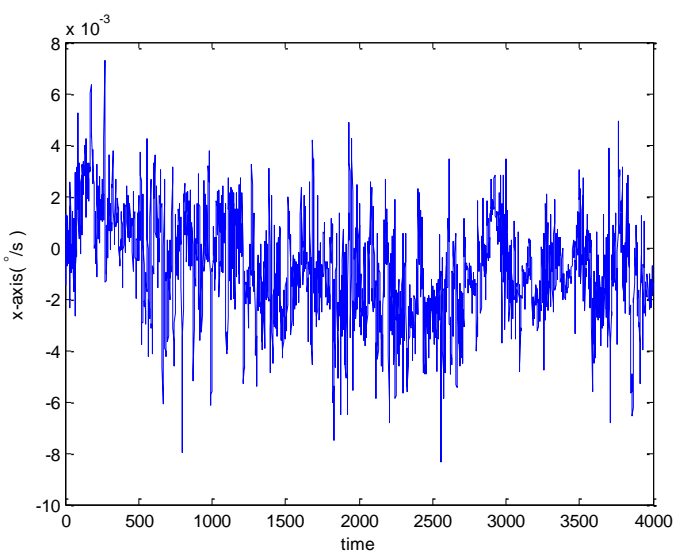

(a)

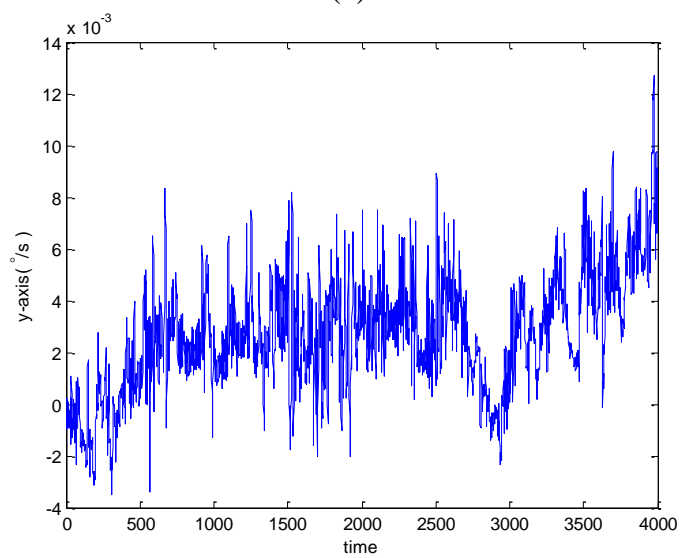

(b) 


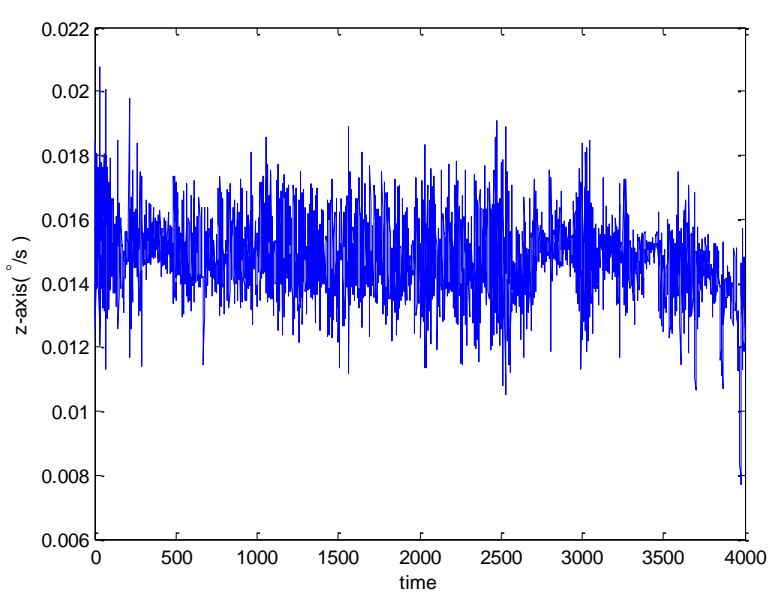

(c)

Figure3. Gyroscope measurements error

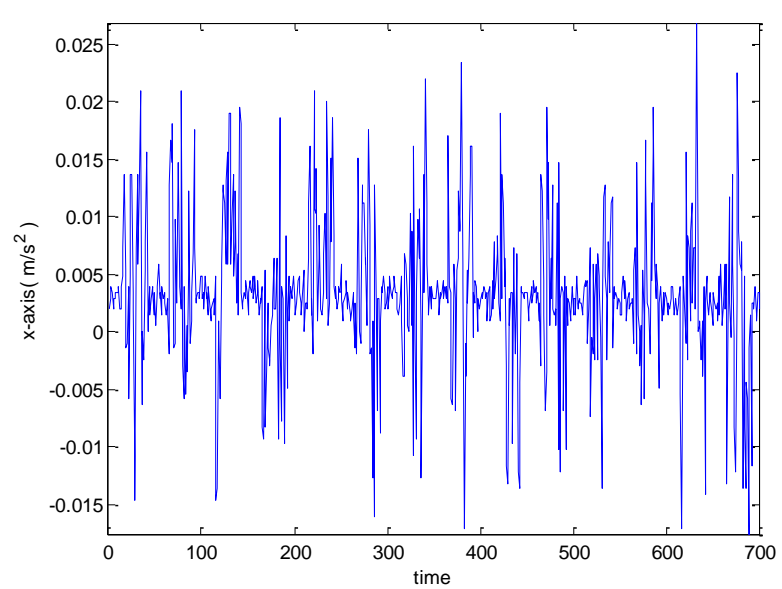

(a)

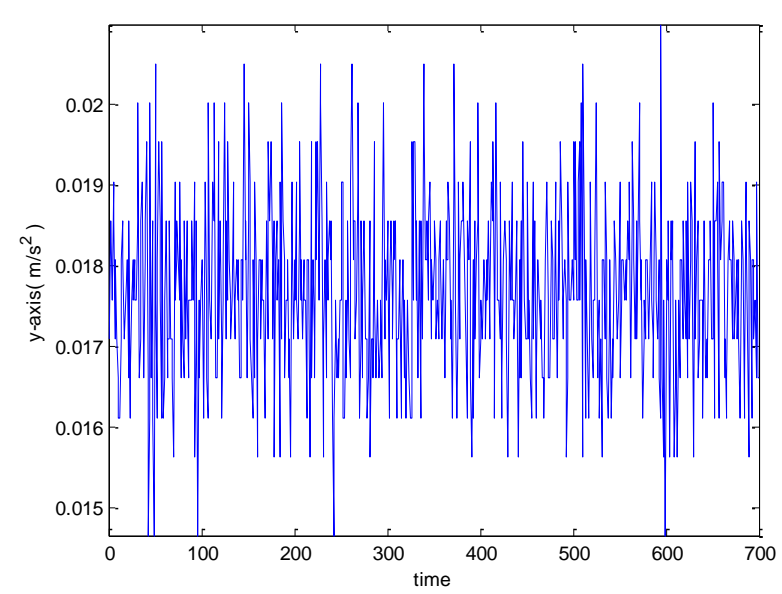

(b)

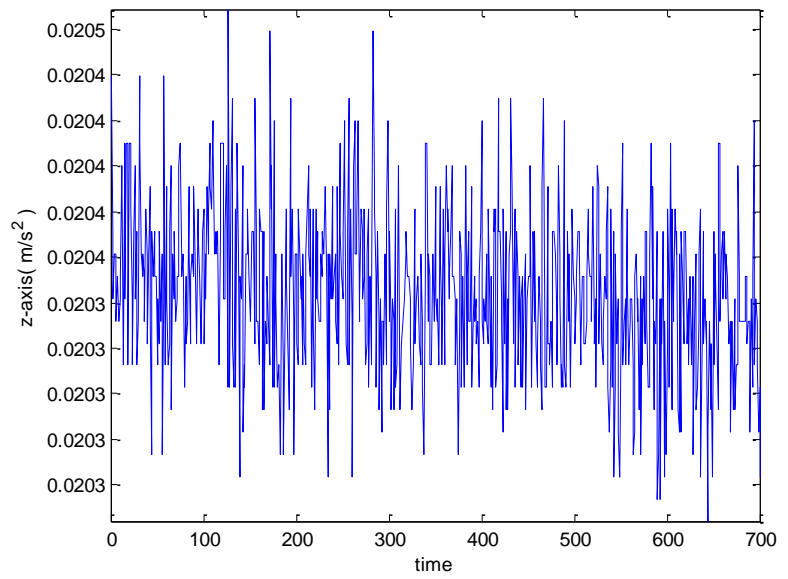

(c)

Figure4. Gyroscope measurements error

\section{CONCLUSIONS}

A systematic numerical analysis on strap-down inertial measurement unit errors is developed. The errors, such as IMU bias errors, IMU misalignment errors, scale factor errors and measurement noise errors, are taken into discussion. Numerical simulations were presented to describe the combined IMU measurements' errors. This work can help to validate the error effect on the performance of the state estimation of the vehicles.

\section{REFERENCES}

[1] W. X. GAO. \& L. J, Miao. 2011. Multiple Fading Factors Kalman Filter for SINS Static Alignment Application. Chinese Journal of Aeronautics 24: 476-483.

[2] Mamoun F. \& Abdel-Hafez. 2011. On the development of an inertial navigation error-budget system. Journal of the Franklin Institute 348: 24-44.

[3] W. X. GAO. \& L. J, Miao. 2001. SINS Static Alignment Application. Chinese Journal of Aeronautics 20(1): 47-53.

[4] Sorenson H W. \& Sacks J E. 1971. Recursive fading memory filtering. Information Sciences. 3(2): 101-119.

[5] Ydstie B. E. T. 1985. Recursive estimation with adaptive divergence control. IEE Proceedings D Control Theory and Applications. 132(3): 124-130.

[6] Qin Y Y.2006. Inertial navigation. Beijing: Science Press.

[7] Sorenson H W. \& Sacks J E. 1971. Recursive fading memory filtering. Information Sciences. 3(2): 101-119. 